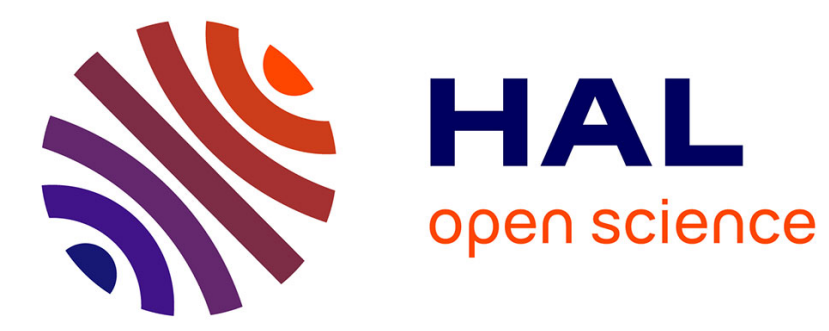

\title{
1789-1830, la nouvelle aristocratie et le peuple: la permanence de la construction de soi par contraste Jacques Guilhaumou
}

\section{To cite this version:}

Jacques Guilhaumou. 1789-1830, la nouvelle aristocratie et le peuple : la permanence de la construction de soi par contraste. Frobert, Ludovic. L'Echo de la fabrique: naissance de la presse ouvrière à Lyon, Ens éditions ; Institut d'histoire du livre, pp.141-160, 2010. halshs-02566946

\section{HAL Id: halshs-02566946 \\ https://shs.hal.science/halshs-02566946}

Submitted on 7 May 2020

HAL is a multi-disciplinary open access archive for the deposit and dissemination of scientific research documents, whether they are published or not. The documents may come from teaching and research institutions in France or abroad, or from public or private research centers.
L'archive ouverte pluridisciplinaire $\mathbf{H A L}$, est destinée au dépôt et à la diffusion de documents scientifiques de niveau recherche, publiés ou non, émanant des établissements d'enseignement et de recherche français ou étrangers, des laboratoires publics ou privés. 


\section{Jacques Guilhaumou}

\section{9-1830, la nouvelle aristocratie et le peuple. La permanence de la construction de soi par contraste.}

Jacques Guilhaumou, «1789-1830, la nouvelle aristocratie et le peuple. La permanence de la construction de soi par contraste », in L'Echo de la fabrique. Naissance de la presse ouvrière à Lyon, sous la dir. de L. Frobert, Lyon, ENSéditions, Institut d'histoire du livre, 2010, p. 141-160.

L'Echo de la Fabrique accorde une grande place à l'usage des désignants de son univers de référence, le peuple, les ouvriers, les prolétaires, etc. et à son espace antonymique, l'aristocratie bourgeoisie ou aristocratie de richesse, qualifiée aussi de nouvelle aristocratie. L'exploration de l'univers des désignants sociopolitiques dans ce journal ouvrier constitue donc, pour l'historien linguiste, un terrain privilégié d'études, mais il peut être abordé de différentes façons. Nous l'étudions présentement sous l'angle de la référence à 1789 , et plus largement à la période des Lumières et de la Révolution française, mentionnée dans de multiples passages. Il s'agit par ce biais historique d'interroger les divers usages de peuple, et surtout d'aristocratie, pour voir dans quelle mesure la désignation d'une nouvelle identité, sous le désignant de peuple (prolétaire), se constitue d'abord par contraste, c'est-à-dire par la mise en place de son antonyme, la nouvelle aristocratie.

Il est tout a fait significatif à cet égard, en ce qui concerne la pérennité de la Révolution française au sein de ce journal, que le portrait d'un prolétaire, Maurice Vichard, dans le $\mathrm{N}^{\circ} 21$ du 18 mars 1832, donc au début de L'Echo de la Fabrique, présente les hauts faits d'un marin avant la Révolution française, devenu l'un des vainqueurs de la Bastille en 1789, puis soldat de la République et des Cent jours, qui sombre ensuite dans la plus absolue pauvreté. Qui plus est, ce portrait est écrit par Marius Chastaing, auteur peut-être le plus représentatif de la sensibilité républicaine de L'Echo de la fabrique, par son esprit pragmatique et réformiste orienté vers l'éducation politique de ses lecteurs ouvriers, comme le montre Jeremy Popkin dans le présent volume.

Ainsi la référence à «la sublime révolution de 1789 » marque toujours l'existence d'un peuple « qui se couvrit de gloire en prenant la Bastille » $\left(\mathrm{N}^{\circ} \mathrm{du}\right.$ 10 février 1833), et à ce titrele peuple demeure le moteur principal de l'émancipation humaine. Mais sans doute fallait-il que l'émergence et les contours de son antonyme, «la nouvelle aristocratie» soient clairement circonscrits pour que ce «peuple prolétaire » atteigne, si l'on peut dire, le plein emploi de sa nomination identitaire. 


\section{I- Critères méthodologiques}

Notre intérêt privilégié, dans la présente étude, pour la resémantisation du couple antonyme aristocrate/peuple, hérité de la Révolution française, dans les expressions nouvelle aristocratie/peuple (prolétaire) tient d'abord au fait que historiens et linguistes ont souligné que, dans les processus de désignation des identités politiques, la désignation péjorée de l'autre est non seulement indissolublement liée à la désignation identitaire, à l'exemple du fameux couple amis/ennemis, mais aussi que cette catégorisation de l'autre précède souvent celle de soi, à l'exemple du couple contre-révolutionnaire/révolutionnaire au début de la Révolution française, étudié par la linguiste Agnès Steuckardt (2002).

Ainsi se précise, au sein du champ sémantique des concepts antonymes asymétriques, une manière spécifique de configurer l'unité d'action des protagonistes de l'événement au niveau conceptuel, comme l'a montré l'historien Reinhart Koselleck (1990). Certes il ne s'agit pas de réduire le mouvement historique à des antagonismes figées et dualistes. Mais les paires conceptuelles antonymes présentent l'avantage de mettre en place une structure d'argumentation permettant de suivre pas à pas le processus de sémantisation des couples opposées, ici aristocratie/peuple. Nous pouvons alors comprendre en quoi l'expérience de l'autre - ici l'expérience de la domination sociale de la nouvelle aristocratie - contribue à stabiliser sémantiquement une figure du peuple qui se prête trop souvent aux jeux d'imprécision des stratégies politiques. Qui plus, au terme de cette étude, nous rejoignons le constat, mis en évidence quantitativement par Maurice Tournier dans le présent volume, que le peuple est lexicalement dominant dans le champ des désignants socio-politiques en 1834, y compris à la fin de la période, au terme du processus que nous étudions.

Au-delà de ce résultat, qui nous intéresse au titre du processus lui-même, se profilent aussi la Révolution de 1848 et sa marque lexicale : "Une évidence s'impose d'entrée de jeu: l'indexation lexicale d'un corpus de textes quarantehuitard (regroupant 192.000 occurrences de mots) fait apparaître peuple en tête de toutes les formes pleines. Si l'on en croit notre échantillonnage, peuple est le terme majeur du discours politique de l'époque. » (Tournier, 1993, 83). Notons également, avec Maurice Tournier, dans le propos conclusif de son étude précitée sur «Le peuple quarantehuitard : un outil de classe » que « ce que veut dire Peuple pendant la révolution de $48:$ une fracture dans la référence, qui s'appelle la lutte des classes » (p. 120). C'est donc aussi de la genèse de la lutte des classes dont il est également question dans notre étude. 
Nous savons en effet, d'après les travaux discursifs de Marie-France Piguet (1996) sur les premiers usages de la lexie lutte(s) de(s) classes, que ce syntagme prend forme avant 1830 dans les écrits des historiens Mignet, Guizot et Thiers. Remarquons aussi que ce syntagme en cours de figement est associé à celui de classe moyenne, plus rarement qualifiée de classe bourgeoise, alors que nous constaterons, dans L'Echo de la Fabrique, un déplacement sémantique vers aristocratie moyenne. De même, nous verrons, dans ce premier journal ouvrier d'envergure, se dessiner une intéressante dialectique entre le fait objectif d'intérêts antagonistes et le principe subjectif qui préside à la formation d'une classe, en particulier la classe ouvrière. Nous y reviendrons donc.

Un dernier point méthodologique concerne la question très classique, trop classique peut-être de l'émergence d'une conscience de classe dans le mouvement ouvrier, tout particulièrement à travers ses premières expressions journalistique et associative. C'est là où les travaux de Gareth Stedman Jones s'avère tout à fait décisif, en particulier son célèbre ouvrage sur Languages of Class : Studies in English Working Classe History (1983) dont nous disposons d'une traduction partielle récente (2007). L'historien anglais précise d'emblée que :

« Nous ne pouvons pas décoder un langage politique pour y découvrir une expression matérielle primitive d'un intérêt, puisque que c'est la structure discursive du langage politique qui crée et définit au départ des intérêts. Nous devons donc étudier la production des intérêts, identifications, revendications, aspirations à l'intérieur des langages politiques eux-mêmes » (p. 21-22)

Ce parti pris langagier, sans pour autant ignorer l'articulation du langage au réel (Guilhaumou 2006, Jones, 2007), est aussi le nôtre. Il s'agit bien de considérer la formulation des intérêts de classe dans leur antagonisme même comme partie intégrante d'un langage du radicalisme, issu en France comme en Angleterre de traditions spécifiques, qui situent alors la question de la conscience de classe, non pas en second rang, mais dans un champ discursif d'expressions radicales particulièrement diversifiées. Au moins sur deux plans. D'une part, cette perspective recoupe l'accent mis sur la dimension pragmatique des constructions sociales au sein de L'Echo de la Fabrique analysé par Ludovic Frobert dans le présent volume. D'autre part, la question demeure de savoir ce qu'il en est de la tradition civique spécifique au contexte lyonnais, dans la production de L'Echo de la Fabrique. Ce second point est délicat dans la mesure où il renvoie au traumatisme de 1793, l'arrestation, voire l'exécution, de nombreux artisans du textile au titre de leur participation au mouvement fédéraliste (Edmonds, 1990), véritable fantôme qui tend à invisibiliser les références pourtant présentes à cette première expérience démocratique révolutionnaire. Nous y reviendrons dans une prochaine étude, soucieux, nous le verrons, de mettre ici plutôt l'accent sur la dimension visible de la référence à la Révolution française, l'héritage de 1789 
en matière d'égalité, tout en terminant sur l'importance, en fin de parcours, de la référence robespierriste, dissociée un temps des excès de la terreur.

Notre présente étude, certes modeste, se situe donc, du point de vue méthodologique, à la croisée d'une histoire du discours contextualisant les arguments au sein même des textes, d'une histoire sémantique s'accordant sur la dimension conceptuelle des usages socio-politiques, et d'une histoire linguistique attentive à la matérialité du discours, et en particulier à sa matérialité lexicale et linguistique. Ce que nous appelons l'histoire langagière des concepts (Guilhaumou, 2006).

\section{II - Portrait d'une nouvelle aristocratie}

\section{Un premier portrait}

Dès le $\mathrm{N}^{\circ} 3$ (1831), le «malheureux peuple » est confronté à un constat, «la puissance n'a fait que changer aux mains des grands du jour». A la haute noblesse, «aristocratie de naissance » succède « une aristocratie de richesse » dont le trait majeur est l'égoïsme. Ce trait caractéristique, que l'on retrouve à diverses reprises, fait même l'objet plus tardivement ( $\mathrm{N}^{\circ} 43$ du 29 juillet 1832), d'un article intitulé De l'égoïsme, repris du journal Le Vulcain, où il est précisé que :

«L'égoïsme est la plaie la plus profonde du corps social, et il ne tend rien moins qu'à le frapper de mort/.../ Il tend à étendre la prédominance absolue de moi /.../ L'égoïste se laisse diriger comme à son insu par cette par cette horrible maxime : la famille, la société, l'état, c'est moi »

Ainsi se positionne une nouvelle aristocratie au plus près du « moi bourgeois », dont Victor Cousin se fera le chantre (Goldstein, 2005), et que l' Echo de la Fabrique dénonce dans «l'esprit d'individualité » qui tend à défaire «le lien politique », et donc transforme le patriotisme en une abstraction.

Dans le $\mathrm{N}^{\circ} 4$ du 20 novembre 1831, une rubrique intitulée «l'aristocratie du comptoir », précise ce mécanisme social d'élévation à une nouvelle grandeur et en notifie les caractéristiques, l'égoïsme certes, mais aussi la duplicité et la trahison au plan historique. Les «hommes du comptoir» qui composent la classe industrieuse apparaissent ainsi sous le jour d'une «nouvelle aristocratie»: s'ils étaient «populaires » et «philanthropes» au départ, lorsqu'il s'agissait de «parler du peuple et pour le peuple» contre "l'aristocratie féodale », de lui promettre l'égalité, il n'en est rien désormais, l'égoïsme l'emporte. De fait, le processus historique se déroule en deux temps : en 1789 , la révolution s'efforce de briser le pouvoir de l'aristocratie féodale au 
profit de «la fortune assise au comptoir » qui, pour en temps, s'associe à l'artisan au nom de l'égalité contre la caste privilégiée : en 1830, la révolution de juillet disperse définitivement l'aristocratie féodale, mais le financier et le commerçant, une fois au pouvoir reconstituent une caste privilégiée, en l'occurrence la nouvelle aristocratie.

Et de revenir sur cette nouvelle aristocratie dans le $\mathrm{N}^{\circ} 22$ du 25 mars 1832 au titre de la désignation d' aristocratie moyenne, sous la plume de Laurent dans une réflexion historique encore plus large sur la manière dont « les vainqueurs du privilège » le déplacent sans le détruire, conférant ainsi à leur participation à la révolution de 1789 un caractère à la fois superficiel et surtout incomplet :

«Partout, dans la lutte incessante de la liberté humaine contre le privilège et l'oppression, nous voyons apparaître, aux grandes époques d'affranchissement l'aristocratie moyenne, qui sait si bien invoquer les lumières du siècle, les bienfaits de la civilisation, l'esprit philosophique, le génie du progrès, pour faire descendre à son niveau tout ce qui le domine, de par la tradition et le préjugé, et qui oublie ensuite sa kirielle libérale, pour ne plus parler que du danger des innovations et de son besoin de repos, d'ordre et de stabilité, dès que le désir d'élévation et l'amour de l'égalité se manifestent au-dessous d'elle»

Et de conclure : «L'apparition périodique de ce phénomène social, dans le développement de l'humanité, est, du reste, facile à expliquer ».

Ainsi il s'agit de caractériser, sous la forme d'une quasi-loi, la récurrence d'un phénomène social dans le développement de l'humanité : le parvenu moderne, à l'exemple de la bourgeoisie défendant la cause du tiers-Etat contre la noblesse au début de la Révolution française, se sépare socialement de «la classe innombrable des prolétaires », et lui demande, de surcroît, d' "interrompre son œuvre d'émancipation ». Les «tribuns de 89 », les Mirabeau, les Robespierre, sont éclipsés : à l'exemple de Rome, tout commence par les Gracques et se termine par César.

Un tel portrait de la nouvelle aristocratie, mis en place au cours de la première période de L'Echo de la fabrique, repose essentiellement sur le fait du partage de la société en deux classes, les prolétaires et « les hommes de la propriété ». Il s'agit alors de convaincre les riches égoïstes qu'ils feraient mieux d'œuvrer pour défendre «les intérêts de la classe ouvrière », dont de contribuer à l'amélioration de son misérable sort plutôt que de « faire de l'aristocratie ». 


\section{En amont de Marx}

Avec Marius Chastaing, auteur du portrait de Vichard le prolétaire, dont nous avons fait mention en introduction, et la seconde période de $L$ 'Echo qui s'ouvre à mi-chemin de l'année 1832, la construction de l'identité du peuple par contraste avec l'aristocratie s'inscrit plus nettement dans ce que qualifions usuellement de lutte des classes. Il s'agit non seulement de souligner l'actualité toujours aussi brûlante de l'appel au peuple au nom du "principe social de l'égalité » contre les privilèges dans une référence toujours aussi appuyée à 1789 tout en marquant les limites sociales des «révolutions accomplies ». Nous y reviendrons. Mais surtout, il importe de faire le constat du caractère distinct « des intérêts classés dans la société » introduit un autre principe, "le principe d'exception et de privilège » au fondement de la lutte des intérêts de classe, comme le propose Anselme Pétetin ( $\mathrm{N}^{\circ} 52$ du 21 octobre 1832) :

«Dès qu'il a été constaté que des intérêts classés dans la société sont distincts, il faut qu'ils soient hostiles; dès qu'ils sont hostiles, ils doivent se combattre jusqu'à ce que l'un ou l'autre succombe; et celui qui doit succomber, c'est celui du petit nombre, c'est le principe d'exception et de privilège que l'histoire nous montre déclinant toujours, depuis l'esclavage antique et le servage féodal, jusqu'au prolétarisme contemporain. Mais ne demandez pas à une aristocratie de s'abdiquer elle-même et de se suicider par peur du combat : ce serait lui demander plus qu'elle ne peut faire, car elle n'existerait déjà plus, si elle était ainsi résignée d'avance à mourir. Il faut qu'un principe, c'est-à-dire une classe, croie à son éternité, et qu'il agisse en conséquence. »

Le principe d'exception et de privilège, propre au petit nombre fait ainsi contraste avec le principe de généralité et d'égalité, qui définit le plus grand nombre : chacun correspond à une classe, la nouvelle aristocratie d'un côté, le peuple prolétaire de l'autre. Telle est donc la loi du progrès social : deux forces, deux principes constituent le mode de subjectivation, sous la modalité de la classe, pour conférer un contenu à la nature des choses, au fait d'intérêts antagonistes. Et le propre de l'aristocratie de la propriété, c'est son aveuglement sur elle-même dans la mesure où «elle semble méconnaître la loi progressive de l'humanité ; car elle s'oppose avec une opiniâtreté inflexible à l'avènement des prolétaires capables à la vie politique qu'ils réclament » $\left(\mathrm{N}^{\circ} \mathrm{du}\right.$ 9 juin 1833).

Notons une telle insistance sur la définition subjective de la classe, comme principe, force de développement donc, tout en renvoyant au fait objectif 
d'intérêts antagonistes, nature des choses : «Je crois à la nature des choses ; je crois à la vitalité des principes », précise Petetin, toujours dans le $\mathrm{N}^{\circ}$ du 21 octobre 1832. De surcroît, cette manière de voir permet de mieux apprécier ce qu'il en est du jugement moral sur l'aristocratie sociale et politique. Il ne s'agit plus d'attirer exclusivement l'attention sur la cruauté de tous temps de cette aristocratie à l'égard des prolétaires («Je ne crois donc pas à la cruauté des aristocrates d'argent» écrit encore Petetin), dans la mesure où sa «participation aux luttes libérales contre les aristocrates de naissance » en fait des individus aux «sentiments très humains ». Il s'agit plutôt de mettre l'accent sur la méconnaissance par la nouvelle aristocratie des lois morales, facteur de progrès social, et c'est à ce titre qu'il est désormais question avant tout du principe comme ressort de la « la nature des choses ». Ce qui confère, à chaque époque historique, à une classe, ici désignée la nouvelle aristocratie, le rôle de frein au progrès social, et à l'autre classe, conceptualisée présentement sous le terme de prolétarisme contemporain, le rôle émancipateur au titre de la lutte, donc d' " un antagonisme qui est le fait lui-même ».

Nous pouvons d'autant mieux apprécier l'importance d'une telle théorisation du prolétarisme contemporain à travers le couple principe (classe)/fait (antagonisme) qu'elle est l'objet, sur le versant philosophique, d'une « traduction caractérisante » chez le Jeune Marx au moment où il rédige en 1844 La Sainte Famille, alors qu'il est présent à Paris et lit de nombreux textes de la Révolution française et des porte-parole du mouvement ouvrier.

La principale cible de Marx est le courant jeune hégélien s'exerçant à la critique de la langue française des penseurs ouvriers, en particulier Proudhon. Contre les abstractions de ces philosophes allemands, il met en valeur, sous la figure du «Proudhon réel» qui s'en tient «à la pratique française de la Masse » entendons le peuple en action, "les revendications relevant de la pratique immédiate », donc une dimension proprement pragmatique, associée aux jugements, même incomplets des hommes agissants et souffrants, ceux qu'on appelle les émeutiers. D'autant que ces jugements « renferment une somme de vérités qui suffisent pour un certain nombre d'inductions comme pour une sphère déterminée de la vie pratique » (La Sainte Famille, p. 35).

Marx en vient ainsi, toujours au plus près de « la langue française » des porteparole de la Révolution française (des révolutionnaires des années 1790 aux "émeutiers 》 des années 1830), à critiquer aussi l'idée que l'homme de la Masse est pris dans des représentations idéologiques, induisant de fausses vérités, donc véhicule une vision erronée des rapports sociaux. La question n'est pas de savoir ce que le prolétariat se représente faussement par le fait de la domination bourgeoise, mais comment il se reconnaît dans l'action, et se construit donc une conscience pratique, agissante.

Marx ajoute alors que «Le Proudhon de la masse trouve que le principe faussé est notre fausse manière de l'entendre, mais qu'il est vrai dans son objet » (id., 
p.37). C'est dire autrement que la Masse se confronte bien à la réalité comme objet, en l'occurrence son existence en tant que classe prise dans un antagonisme. Ainsi le principe de classe se formule, du fait de l'antagonisme, dans les revendications très matérielles de justice, et plus largement d'égalité, étant entendu que «la formule, c'est le principe en tant que principe de développement scientifique » (id., p. 38). Traduisons que les revendications pratiques de la classe prolétaire procèdent de son propre mouvement, de sa propre réalité scientifique.

Analysant ce moment marxien de la découverte à la fois pratique et philosophique de «l'essence » du prolétariat dans La Sainte Famille, Georges Labica (1976) ajoute que si le prolétariat est, en tant que classe, le principe de son propre mouvement, par le fait même de l'antagonisme dont il est le principal élément agissant, c'est donc bien en lui-même qu'il trouve les conditions du progrès social, et détermine ce qu'il va advenir de la société dans un stade supérieur. «Parler français », comme le fait ainsi Marx, et nulle part de façon si systématique que dans La Sainte Famille, s'avère donc la condition majeure de la manière absolue de définir l'égalité : "L'égalité, c'est la conscience que l'homme a de lui-même dans le domaine de la pratique, c'est-à-dire, par conséquent, la conscience qu'un homme a d'un autre homme comme étant son égal et le comportement de l'homme à l'égard d'un autre homme comme vis-àvis de son égal » (id., p. 50). Et Marx de conclure : «L'égalité est l'expression française pour traduire l'unité essentielle de l'être humain », dans une extrême proximité avec la Révolution française et le mouvement ouvrier.

\section{Autour de Sieyès}

Tout cela relève bien en fin de compte d' « une loi de conservation providentielle». Prolétaires et bourgeois sont ainsi confrontés à «une loi de conservation politique » facteur de progrès social qui s'exemplifie, dans la période contemporaine, après la mort de la noblesse féodale, la disparition de « la classe des nobles » et la mise en visibilité de « la classe bourgeoise »:

«La classe de citoyens ayant ce quelque chose de visible, de palpable, auquel la loi attache une prérogative politique, cette classe de bourgeois est fort peu nombreuse; elle forme réellement une aristocratie tout aussi concentrée que la noblesse de l'ancien régime, et tout aussi jalouse de ses privilèges » $\left(\mathrm{N}^{\circ} \mathrm{du} 27\right.$ janvier 1833, extrait de Le National).

A la visibilité politique extrême de la bourgeoisie, par le fait de ses privilèges, répond donc l'invisibilité du peuple prolétaire. Mais, en contrepartie, à l'aveuglement de la bourgeoisie sur son avenir correspond la prise de conscience du prolétariat de son rôle émancipateur dans l'histoire. 
Ainsi, là où les bourgeois ne « conçoivent pas d'autre moyen d'avoir pour tous que celui de prendre à quelqu'un », les prolétaires réitèrent la formule du TiersEtat : «Qu'est-ce que le tiers état - TOUT / Qu'a-t-il été jusqu'à présent dans l'ordre politique - RIEN / Que demande-t-il - A ETRE QUELQUE CHOSE ». Et l'auteur de cette référence connue à Sieyès, mais non cité, d'ajouter : "Qu'à la place du mot tiers-état on mette le mot prolétaire, et l'on trouvera que ces questions sont encore à l'ordre du jour. Or, on se souvient de ce qu'il advint lorsqu'elles furent posées pour la première fois ».

Cette référence au texte princeps de Sieyès ouvrant Qu'est-ce que le Tiers-Etat? se trouve dans un article de C.B. du $\mathrm{N}^{\circ} 23$ du 9 juin 1833 aux accents, il est vrai, fortement sieyèsiens. L'accent y est mis en effet sur « la nouvelle aristocratie qui domine aujourd'hui » et sur le constat, qu'elle est «bien une petite minorité dans la nation », « un petit nombre de privilégiés de la fortune ». Sieyès considérait que les privilégiés de son temps s'excluaient de la nation en se mettant hors du commun. En 1833, ces privilégiés sont les seuls éligibles, donc ils sont de fait fortement intégrés dans la nation. S'il convient certes de dénoncer cette exclusivité, établie au détriment des droits politiques des prolétaires, ni éligibles, ni électeurs, il n'est question de donner ces droits qu'aux « prolétaires capables », "hommes instruits et éclairés », élargissant ainsi le cercle des citoyens à part entière. C'est donc plutôt de « la loi progressive de l'humanité », de son caractère naturel que l'aristocratie s'exclue, au risque, par son petit nombre, de disparaître, à la différence des privilégiés de 1789 perçu par Sieyès comme hors du commun, de la nation. Nous sommes donc moins dans la construction désormais effective du «tout de la nation» (Sieyès) que dans la dynamique de la progressivité de la nation dont les bourgeois privilégiés, à distinguer de la classe moyenne, "plus proche de la classe ouvrière que de l'aristocratie bourgeoise ", s'excluent par leur égoïsme. Mais l'invoncation générale demeure de facture sieyèsienne.

Ainsi l'aristocratie n'est pas naturelle. L'invocation « Aux aristocrates d'aujourd'hui », à laquelle s'ajoute une suite «Encore aux aristocrates d'aujourd'hui » ( $\mathrm{N}^{\circ}$ du 28 avril et 12 mai 1833), complètent alors le portrait antinaturel de la nouvelle aristocratie, et de sa «résistance impie à la loi du progrès ». Ainsi, un univers bien contrasté par la figure mouvante de l'aristocratie permet de définir l'actualité d'une figure du peuple formulée démocratiquement dès 1789 , et à l'horizon d'une humanité qui "marche incessamment vers un avenir d'amélioration »(Jullien, L'humanité marche, Numéro du 10 février 1833).

Une contextualisation plus large, comme le propose Lucien Jaume lorsqu'il constate qu' " une notion du $19^{\text {ème }}$ siècle français devrait attirer davantage les historiens de la pensée politique: en effet, entre 1800 et 1880, 
approximativement, le débat sur les institutions appropriées pour la société issue de la Révolution française roule en grande partie sur la nouvelle aristocratie, groupe social à la fois attendu, recherché et introuvable » (2006, p. 969), permet également de mieux comprendre l'enjeu d'une telle référence ouvrière au caractère antinaturelle de la nouvelle aristocratie.

En se tournant vers les penseurs libéraux de la période, et en particulier les doctrinaires, comme le fait Lucien Jaume, il apparaît qu'au-delà des sens divers attachés à la nouvelle aristocratie - entre la classe des chevaliers d'industrie et la classe moyenne, en passant par la classe politique -, un volonté de naturaliser l'aristocratie, donc de promouvoir la classe gouvernante selon l'expression de la véritable, la naturelle aristocratie de l'ordre nouveau se précise. Ainsi, l'auteur de la précédente expression, Guizot justifie le fait anthropologique et sociologique de l'aristocratie par sa supériorité - ici le droit ne se réduit pas au fait, mais le droit naît à partir du fait - et en déduit des intérêts communs avec le peuple dans les termes suivants :

«Quand les vrais avantages que recherchait le peuple en réclamant l'égalité sont obtenus, les classes inférieures elles-mêmes s'apaisent /... La nouvelle aristocratie qui s'est formée à des intérêts qui leur sont communs et les protège en les défendant. Elle contient le peuple par les liens qui l'unissent à lui. Alors l'idée de l'égalité apparaît sous une forme plus calme et plus dure » (Des moyens de gouvernement et d'opposition, 1821, cité par Jaune, 2006, p. 972).

Nous pouvons ici mesurer toute la distance qui sépare les porte-parole républicains et ouvriers, dans L'Echo de la Fabrique, des penseurs libéraux doctrinaires. C'est d'ailleurs sur le terrain même d'un certain pragmatisme, donc dans une dimension très pratique, que l'incapacité de la nouvelle aristocratie à suivre la loi naturelle de l'humanité, par son caractère foncièrement oligarchique, devient visible. De «l'idée d'égalité »(Guizot), il est donc aussi question chez ces porte-parole, mais dans un sens bien différent.

\section{II - 1789 - 1830 : de l'égalité politique à l'égalité sociale au sein du peuple.}

Tout commence, on s'en doute, par une mise en évidence de la limite du " principe de l'égalité politique » tel qu' «il a été conquis en 1789 ». Dans le №30 du 20 mai 1832, Marius Chastaing signe un article intitulé De l'égalité sociale. Il y est énoncé que «les tribuns de 1789 » ont établi l'égalité politique sur la ligne d'un progrès dont la prochaine étape est « l'adoption d'un nouveau principe », celui de l'égalité sociale, après « la révolution de juillet 1830 ». Il annonce la seconde période du journal où l'accent est mis sur les limites sociales des révolutions antérieures. 
Ainsi, dans le $\mathrm{N}^{\circ} 36$ du $1^{\mathrm{er}}$ juillet 1832 , il fait part de sa «conviction profonde » en la matière :

«Il n'y a de révolutions durables et fécondes, de révolutions accomplies sans retour, que celle qui intéressent directement la classe nombreuse et laborieuse, qui améliorent radicalement sa condition morale et matérielle. Le peuple ne gagne rien effectivement aux mouvements politiques qui n'ont d'autre résultat que celui de transporter le pouvoir d'une fraction à une autre de la classe privilégiée. Les distinctions naguère encore si profondes de nobles et de bourgeois, de monarchistes et de libéraux, de wigs et de torys ont été effacées par l'apparition d'un nouvel élément demeuré pour ainsi dire inaperçu jusque là, LE PEUPLE ».

La figure du peuple devenu prolétariat se resémantise donc au départ par contraste avec la bourgeoisie, au titre de la nouvelle aristocratie. Elle devient l'élément central d'une loi du progrès humain qui a valeur de religion sociale. C'est là où s'imposent la figure tutélaire de Condorcet et le rôle majeur de « son livre de la perfectibilité » (il s'agit bien sûr du Tableau historique des progrès de l'esprit humain). Marius Chastaing peut alors énoncer sa foi dans la «loi du progrès » et son but «dans un avenir peu éloigné, l'émancipation de la classe prolétaire », dans un mouvement incluant les Lumières et la Révolution française :

«Nous savons aussi que dans une période de paix, toutes les idées dont les germes commencent à poindre se développeront malgré les efforts d'un despotisme pygmée, et formeront en peu de temps une philosophie du $19^{\text {ème }}$ siècle qui amènera la grande rénovation sociale dont la révolution de 89 , fille de la philosophie du $18^{\text {ème }}$ siècle, n'a été que le prélude ».

Une même tonalité sur la loi du progrès social depuis Condorcet et la Révolution française se retrouve dans l'ensemble de L'Echo de la Fabrique, ainsi par exemple au sein de la rubrique, déjà citée, «L'humanité marche » dans le numéro du 17 février 1833 :

«Nous retrouvons partout cet accord unanime des esprits élevés à reconnaître dans ce qui se passe depuis quarante ans, et dans tout ce qui s'accomplit autour de nous, un signe évident de transformation sociale /../ L'humanité marche incessamment vers un avenir d'amélioration : malheur à ceux qui sont assez présomptueux, assez aveugles pour se croire pouvoir d'arrêter ce torrent des siècles ! » 
Et l'auteur, Jullien, de faire référence au génie prophétique de Chateaubriand, voire de le citer : "L'on ne cesse de s'étonner des événements, toujours on se figure atteindre le dernier, toujours la révolution recommence ».

Une telle logique sociale de révolution permanente pousse certains auteurs de L'Echo de la Fabrique, en particulier Bouvery en plein débat avec Pétetin (voir ci-après), a mettre en cause, une fois rappelé que «les Voltaire, les Rousseau, les d'Alembert, les Diderot, etc., sont venus révéler au monde la monstrueuse organisation sociale du genre humain en général » ( $\mathrm{N}^{\circ} 40$ du 19 août 1832), la manière dont « la révolution de 89 » impose l'ordre politique à l'ordre matériel, alors qu'il convient d'opérer l'inverse, en marchant «dans la voie des améliorations matérielles » avec ceux qui souffrent le plus de l'ordre existant, les prolétaires. Une même réflexion introduit à la «leçon grammaticale », rubrique du $\mathrm{N}^{\circ}$ du 31 mars 1833, sur les usages abusifs du mot Monsieur :

«Que vous êtes sots, vous autres qui croyez que 1789 et 1830 ont aboli les distinctions sociales dont la vanité des hommes est si avide, détrompez vous, elles n'ont fait que changer de nom, elles existent aujourd'hui tout aussi vivaces que dans les temps féodaux les plus reculés »

Le peuple reste donc toujours et encore la figure à resémantiser comme le montre la rubrique LE PEUPLE sous la plume de Miran, dans le numéro du 10 février 1833. Là encore, comme l'avait fait Chastaing, il s'agit d'abord d'écarter l'épouvantail dressé par les bourgeois à partir de l'image des hommes de 1793 :

«Le peuple n'est point cet être aveugle qui frappe sans examen. On nous cite 93 ; mais ce n'est pas le peuple qui frappait alors, c'était l'étranger qui soudoyait des hommes féroces, qui avaient mission de déconsidérer le peuple et sa sublime révolution de 89 »

Mais l'essentiel demeure dans la difficulté même à stabiliser sémantiquement le désignant de peuple :

«On entend chaque jour de mot avec un étrange et ridicule dédain, le peuple !...Et chaque individu qui l'emploie prétend ne pas appartenir à la catégorie qui le désigne. C'est pitié de voir cet orgueil, ce dédain comique pour la plus noble désignation »

De ce rapide tour d'horizon des désignants d'aristocratie et de peuple, nous pouvons conclure que la nouvelle aristocratie, présente sous diverses désignations (nous y reviendrons ultérieurement dans une analyse lexicale des 
concordances), apparaît sémantiquement et lexicalement plutôt stable, dès le début du journal. Elle s'inscrit, si l'on peut dire, dans un fonds républicain relativement constant, même s'il est surtout entretenu par les auteurs les plus proches de l'héritage républicain de la Révolution française, en particulier Marius Chastaing, et donc moins aptes à traduire l'expérience de la classe ouvrière, même s'ils ont une étonnante capacité aux anticipations sociales.

Le débat entre Bouvery et Pétetin, à l'automne 1832, montre bien la portée et les limites de l'apport des journalistes républicains de L'Echo de la fabrique. Dans l'étude que nous en proposent Alain Clément et Ludovic Frobert dans le présent ouvrage, il apparaît que la voix de Bouvery, chef d'atelier mutuelliste et porte-parole des canuts, incarne la figure sociale du peuple par le fait d'un espace lexical traduisant l'expérience des conditions de travail acquises dans la négociation, voir la lutte, ce qui nous situe bien au-delà de la description discursive de l'opposition aristocratie/peuple que nous venons de mener à bien. Son intervention d'orientation fortement pragmatique fait donc singulièrement contraste avec le discours conceptuel de Pétetin plutôt orienté vers des questions d'organisation sociale et de système de gouvernement adéquat à « l'amélioration du sort moral et matériel des travailleurs ». Bouvery se présente en effet comme un «prolétaire », certes sensible au débat avec les «hommes éclairés », mais plutôt réservé sur leur manière de considérer ce qui doit être, et non ce qui est, donc trop centrés sur ce qui relève de l'artifice politique des institutions républicaines. Il recherche plutôt l'adéquation des choses aux idées au sein même de l'expérimentation sociale et politique, en particulier dans le cadre coopératif, mutuelliste.

C'est donc bien que la figure du peuple ne se résémantise pas, à l'horizon de l'émancipation humaine, sans un combat, une lutte dont les mots sont partie prenante. Elle concerne l'ensemble des sensibilités du journal, et aussi les auteurs les plus proches des canuts. La construction de soi de la classe inférieure des prolétaires n'a rien d'un parcours facile, donc simplement énonçable abstraitement. A ce titre, elle met en œuvre une analyse conceptuelle qui, entre le principe, la classe, et le fait, la lutte constitue à sa manière une théorisation de la lutte des classes. C'est là où le fait social s'ancre dans l'intentionnalité, une intentionnalité collective partagée par tous et formulée dans les termes de l'association. Cependant, il convenait, dans une première étape, de (re) formuler la part héritée d'intentionnalité individuelle dont les républicains demeurent les garants auprès des ouvriers, et qui se construit dans la structure binaire aristocratie/peuple.

Nous en venons alors à considérer que, dans L'Echo de la Fabrique, une fois formulés les éléments conceptuels d'une ontologie sociale propice à la formation discursive de la lutte des classes, il peut se formuler un attitude plutôt modérée. 
Ainsi dans le dernier numéro du 4 mai 1834, il y est reproduit le bulletin de souscription d'un autre journal, le Peuple Souverain, où il est précisé : "Nous avons assez souvent dit que ce n'est point d'une lutte permanente entre les différentes classes qui composent la grande famille sociale que doit sortir le triomphe de la cause des travailleurs ». A vrai dire, une fois mis en place les éléments, le principe et le fait, propices à la génération discursive de l'expression de la lutte des classes, cette expression peut devenir l'enjeu d'un débat. Elle n'est pas la réification d'une conscience de classe : l'affaire est plus complexe, dans la mesure où elle partie prenante des enjeux langagiers mis en œuvre dans son emploi. A ce titre, les intérêts divers des rédacteurs de L'Echo de la Fabrique s'organisent d'abord dans une certaine dynamique du langage politique où l'opposition aristocratie/peuple joue un rôle fondateur, avant même que se précise leur diversité idéologique, dans leur relation plus ou moins proche avec la classe ouvrière lyonnaise.

De cette diversité, nous retiendrons, dans notre propos conclusif, la référence attendue à Robespierre, et sa marque dans L'Echo de la Fabrique sans trancher sur son importance au sein de l'équipe des rédacteurs.

En guise de conclusion : de Robespierre à l'Echo de la Fabrique, le projet d' "économie politique populaire»

Dans le $\mathrm{N}^{\circ} 7$ du 17 février 1833, la rubrique «Lectures prolétaires » comprend, pour l'essentiel, des citations de discours de Robespierre en 1793. L'ensemble de ces références est d'une grande cohérence : il précise ce qu'il est du projet robespierriste, défini par lui-même à partir de l'expression d'économie politique populaire. Ainsi il est d'abord question de la loi sociale, dont le principe est la vie elle-même, et dont les moyens - les moyens de la société - sont centrés autour de la mise en œuvre du droit à l'existence. Ce qui confère comme but à la société, la conservation des droits de l'homme, au titre de la première loi sociale, le droit à l'existence, et par conséquent la subordination de la propriété au droit à la vie.

L'historienne Florence Gauthier (1988) a précisé toute l'ampleur de ce projet robespierriste dans la perspective du libéralisme de droit naturel et sous le terme d'économie politique populaire. Il s'agit bien d' «un projet de démocratie économique, sociale et politique, et un type de développement économique alternatif au processus d'expropriation-prolétarisation en cours, se proposant de résoudre le problème crucial du droit à l'existence », là où «le but de l'association politique est d'assurer le maintien des droits naturels à l'existence et à la liberté, et le développement de toutes les facultés de l'homme» (p. 136). Un projet de société qui a donc été pensé, par les Montagnards et nommé par Robespierre au moment de la Révolution française, donc qui a été mis en œuvre par la Convention Nationale en 1793-an II. 
Un article de Trélat, dans le numéro du 7 juillet 1793, explicite cet héritage révolutionnaire. Après avoir débuté par la formule «Pourquoi sommes-nous en révolution, jusqu'à quand serons nous en révolution.. », il est fait référence à la Convention nationale, « l'immortelle assemblée qui seule osa toucher du doigt la plaie qui dévorait notre organisation sociale », en déclarant que «Le société est obligé de pourvoir à la subsistance de tous ces membres, soit en leur procurant du travail, soit en assurant les moyens d'exister à ceux qui sont hors d'état de travailler », d'où le mot d'ordre lui aussi pensé dans la lignée de la Révolution française, «il faut aux classes pauvres du travail et du pain», ou tout simplement « du travail et du pain ».

Ludovic Frobert, dans son ouvrage sur L'économie politique populaire de la Fabrique, titre provisoire sous sa première forme manuscrite, reprenait l'expression robespierriste en l'associant au cri de ralliement des canuts en insurrection, «Vivre en travaillant ou mourir en combattant!» qu'ils avaient inscrits sur leur bannière. Avoir du pain, avoir accès à l'éducation et à la santé, refuser l'oppression de l'arbitraire, disposer de lumières, de connaissances, etc., tels sont les élément sociaux que les canuts revendiquent dans une étroite relation à la politique de la Convention sociale en l'an II. Ici la référence à la terreur de 1793, certes présente nous l'avons vu, s'estompe. Il s'agit beaucoup plus, dans la référence à Robespierre, de marquer ce qu'il peut en être d'un projet permettant d'instaurer le principe (social) d'égalité sociale dont nous avons montré la centralité dans le langage républicain, et ouvrier en fin de parcours, de l'Echo de la Fabrique.

Et de surcroît, la dernière citation de Robespierre, dans la rubrique «Lectures prolétaires " porte de nouveau sur l'égoïsme de tous les temps de l'aristocratie, et nous renvoie ainsi à la pérennité d'une approche de l'aristocratie mise en place dès les premiers numéros de L'Echo de la Fabrique. Terminons donc par cette citation de Robespierre :

«Depuis Jésus les griefs de l'aristocratie n'ont pas varié d'un mot. Quand les apôtres prêchèrent au nom de leur maître crucifié la fraternité universelle, les aristocrates de tous les pays bafouèrent le dévouement de Jésus ; sa croix fut une folie aux yeux de leur égoïsme ; scandalum crucis. Le peuple seul partagea cette folie. Nous voulons un ordre de choses où toutes les passions basses et cruelles soient enchaînées, toutes les passions généreuses et bienfaisantes éveillées par les lois...Nous voulons, dans notre pays, substituer la morale à l'égoïsme, le mépris du vice au mépris du malheur...»

\section{Références bibliographiques}


Edmonds W.D. (1990), Jacobinism and the Revolt of Lyon (1789-1793), Oxford, Oxford Clarendon Press.

Gauthier Florence (1988), «De Mably à Robespierre. De la critique de l'économique à la critique de la politique », in E.P. Thompson et alii., La guerre du blé au XVIIIème siècle, Paris, Les Editions de la Passion, p. 111-144.

Goldstein Jan (2005), The Post-Revolutionary Self: Politics and Psyche in France, 1750-1850. Cambridge, Mass: Harvard University Press.

Guilhaumou Jacques (2006), Discours et événement. L'histoire langagière des concepts, Presses Universitaires de Franche-Comté.

Jaume Lucien (2006), « Tocqueville face au thème de la 'nouvelle aristocratie'. La difficile naissance des partis en France», Revue française de science politique, v. 56, $\mathrm{N}^{\circ} 6$, décembre 2006, p. 969-984.

Koselleck Reinhart (1990), Le futur passé. Contribution à la sémantique des temps passés, Paris, Editions de l'EHESS.

Labica Georges (1976), Le statut marxiste de la philosophie, Bruxelles, Editions Complexe?

Marx Karl (1969), La Sainte Famille (1844), Paris, Les Editions sociales.

Piguet Marie-France (1996), Classe. Histoire du mot et genèse du concept. Des Physiocrates aux Historiens de la Restauration, Presses Universitaires de Lyon.

Stedman Jones Gareth (1983), Languages of Class : Studies in English Working Classe History, 1832-1982, CUP.

Stedman Jones Gareth (2007), «Repenser le chartisme», Revue d'histoire moderne et contemporaine, 54-1, $\mathrm{p}$ ?

Steuckardt Agnès (2002), "Les ennemis selon L'Ami du peuple, ou la catégorisation identitaire par contraste », Mots, $\mathrm{N}^{\circ} 69$, p. 7-22.

Tournier Maurice (1993), Des mots sur la grève. Propos d'étymologie sociale, volume 1, Paris, Klincksieck.

Annexe 1 
De la fréquence aux contextes: vue d'ensemble sur les concordances d'aristocratie et de sa série lexicale.

Si l'on examine les 118 occurrences de la série lexicale, aristocratie, aristocrates, artistocrate, aristocratique, avec une nette dominance d'aristocratie ( 70 occurrences), on constate d'abord une répartition très déséquilibrée dans le corpus d'ensemble, même en considérant le poids relatif des parties: 9 attestations en 1831, 28 en 1832, 58 en 1833, puis une brusque chute, 1 ( l'orgueil aristocrate) en 1834. Le contraste est d'autant plus frappant par comparaison avec la répartition de peuple, et surtout ses occurrences en 1834 : 34 en 1831, 288 en 1832, 336 en 1833, et encore 153 en 1834. Au plan quantitatif, la stabilisation progressive du sémantisme d'aristocratie favorise bien une resémantisation de peuple par contraste en fin de parcours.

En terme de fréquence d'aristocratie d'un article à l'autre, l'article leader est celui signé C.B, et intitulé «Du progrès social» de 1833 : il est argumenté autour de l'expression « la nouvelle aristocratie qui domine aujourd'hui est une bien petite minorité dans la nation ». Ainsi sur 9 occurrences d'aristocratie, on en trouve 5 pour aristocratie bourgeoise (dont une aristocratie financière et bourgeoise). En refusant «l'adjonction des capacités», en s'octroyant l'exclusivité politique sur la base «des privilèges de la naissance et de la fortune » l'aristocratie bourgeoise rapproche la classe moyenne de la classe ouvrière dans leur demande conjointe du « droit à l'émancipation politique », en particulier sur le plan électoral.

C'est ici le terme d'une évolution sémantique précédée en 1831, sous la plume de Vidal, dans un article intitulé «l'aristocratie du comptoir» avec 5 occurrences, par la désignation inaugurale de la nouvelle aristocratie, appréhendée dans une évolution historique : l'aristocratie féodale «tombe en décrépitude », en trouvant sa rivale dans « la fortune assise au comptoir». «Les hommes qui composaient la nouvelle aristocratie »' appuie d'abord sur le peuple pour conquérir le pouvoir, puis se refuse à toute réforme face à « la fermentation politique $\gg$ du peuple.

Vient ensuite un article de Pétetin en 1832 également avec 5 occurrences (12 pour l'ensemble de la série lexicale), qui part d'abord du constat, dans un texte préalable au rédacteur de l'Echo que «le principe aristocratique qui gouverne l'Angleterre a envahi jusqu'à l'industrie », thème déjà développé par François Barthélemy Arlès-Dufour dans un article antérieur, mais de la même année, sur "les privilèges qui font la puissance et l'influence de l'aristocratie anglaise » (huit occurrences d'aristocratie dans deux articles d'un mois sur l'autre). Dans son article proprement dit «Des machines dans l'industrie », Pétetin s'interroge sur le positionnement précis de «principe aristocratique » dans le cas français. Il affirme d'abord que «les passions exclusives des aristocrates seront impuissantes contre la volonté générale », à condition que «les fractions du peuple» deviennent solidaires dans «l'association». Il pense également, à la 
différence de Bouvery, qu'il faut compter sur « la générosité des aristocrates » animés de «sentiments très humains » dans la mesure où, à la différence de l'aristocratie anglaise, ils ont participé aux «luttes libérales» pour l'amélioration du sort du peuple ». Et de conclure ; « Je ne crois pas à la cruauté des aristocrates d'argent ». C'est là où « le principe aristocratique » relève non de bons sentiments, mais de « la nature des choses », renvoyant ainsi à la force des intérêts de classe. C'est donc seulement dans le combat pour le principe opposé, « le principe aristocratique », que l'aristocratie sociale et politique peut faire des concessions.

Enfin notons les occurrences nombreuses, mais plus dispersées, de Marius Chastaing, y compris au sein de la série lexicale aristocratie, aristocrate(s), aristocratique. Il emploie 5 fois aristocratie en 1832, et une fois en 1833 dans divers articles, mais surtout pour désigner l'aristocratie nobiliaire, désormais ruinée et agonisante, tout en parlant de l'aristocratie financière et de «la prétendue aristocratie de propriété ». Il en revient donc d'abord au parcours historique de l'aristocratie moyenne qui devient la véritable aristocratie en se mettant un temps à la tête des classes opprimées, mais s'avère incapable d'aller au-delà de cette « révolution incomplète » Quant à la dispersion lexicale, elle se concentre surtout dans la première période, 18 occurrences en 1832 : elle permet de désigner le caractère des aristocrates de toute nature: une disposition aristocratique toute emprunte d'esprit aristocratique, d'ironie aristocratique et de dédain aristocratique. Et de conclure au terme de l'année 1832 : «L'Echo est l'ennemi de toutes les aristocraties, il n'en reconnaît qu'une, celle du talent » (1832-12-16). 